\title{
De la Démocratie en Chine : éssai d'archéologie du discours sinologique
}

Jean-Yves HEURTEBISE

\section{(2) OpenEdition}

\section{Journals}

Édition électronique

URL : http://journals.openedition.org/transtexts/404

DOI : 10.4000/transtexts.404

ISSN : 2105-2549

Éditeur

Gregory B. Lee

Édition imprimée

Date de publication : 7 avril 2011

ISSN : 1771-2084

Référence électronique

Jean-Yves HEURTEBISE, «De la Démocratie en Chine : éssai d'archéologie du discours sinologique », Transtext(e)s Transcultures 跨文本跨文化 [En ligne], 6 | 2011, mis en ligne le 05 avril 2011, consulté le 02 mai 2019. URL : http://journals.openedition.org/transtexts/404 ; DOI : 10.4000/transtexts.404

(c) Tous droits réservés 


\title{
Transtext(e)s Transcultures 跨文本跨 文化
}

Journal of Global Cultural Studies

6 | 2011:

Debating China

\section{De la Démocratie en Chine : éssai d'archéologie du discours sinologique}

\author{
JEAN-YvES HEURTEBISE
}

\section{Résumés}

La « Démocratie », comme forme de participation plurielle de la société à la politique interne de l'Etat impliquant la mise en demeure possible de l'Etat par les membres de la société, est-elle une pratique sociale reflétant des valeurs culturelles spécifiques ou bien une construction politique universelle en droit ? Cette question générale sera traitée sous l'angle particulier des relations intellectuelles entre la Chine et l'Europe de la Renaissance à nos jours (de Matteo Ricci à François Jullien). Notre propos sera d'établir une archéologie philosophique du discours officiel assimilant la Démocratie à une de ses « valeurs occidentales » étrangères à la Chine - en montrant à la fois ses origines européennes (Hegel, Weber...) et ses limites conceptuelles (Blaut, Wolin...) de même que ses conséquences politiques paradoxales (Taiwan). Cet article sera donc l'occasion de retracer une généalogie du discours sur la Chine et d'examiner le lien entre culture et politique dans une perspective comparatiste en questionnant ainsi un des enjeux majeurs du monde Chinois contemporain.

Is Democracy, defined as the interaction between a pluralist society and a governing State of law where the actual representative of the latter have to take into account the rights and the expectations of the actual members of the former, a social practice embedded in cultural values or a political principle universally valid? This problem will be addressed respectively to China \& Europe's intellectual relationships during the last five hundred years. Our aim is to historically and philosophically evaluate the validity of the claim prevailing in Chinese contemporary politics stating that Democracy is a "Western value" foreign from and alienated to both Chinese past culture and China's present society. We will show that this idea comes in the first place from nineteenth century European theories of Culture which emerged in a nationalist 
anti-Enlightenment context and analyze its paradoxical contemporary geopolitical consequences regarding the Taiwanese case. Therefore, dealing with the relationships between Culture and Politics in an Intercultural context will allow us to critically examine the meaning of the concept of Culture while addressing one of the major issues of contemporary China.

\section{Texte intégral}

中國必須立即開始實行下列兩方面的民主改革。第一方面，將政治制度上國民黨一黨派 一階級的反動獨裁政體, 改變為各黨派各階級合作的民主政體。這方面, 應從改變國民 大會的選舉和召集上違反民主的辦法, 實行民主的選舉和保證大會的自由開會做起, 直 到制定真正的民主憲法，召集真正的民主國會，選舉真正的民主政府，執行真正的民主

政策為止。 毛澤東 : 中國共產黨在抗日時期的任務（一九三七年五月三日） ${ }^{1}$

\section{Introduction : la démocratisation est-elle une donnée culturelle?}

Le 8 octobre 2010, le Prix Nobel de la Paix était attribué à Liu Xiaobo (劉曉 波), arrêté le 23 juin 2009 sous le chef d'inculpation "d'incitation à la subversion du pouvoir de l'État », pour avoir été l'un des promoteurs de la Charte 08 (língbāxiànzhāng 零八憲章) demandant la mise en œuvre de la " cinquième modernisation " : la démocratie. ${ }^{2}$ Le lendemain, un article du Global Times (huánqiúshíbào 環球時報) fustigeait le choix du Comité Nobel en l'expliquant par un refus de l'Occident, imbu de ses propres valeurs culturelles, de reconnaître la spécificité chinoise :

Friday the 2010 Nobel Peace Prize was awarded to Liu Xiaobo, an incarcerated Chinese criminal [...] The Nobel committee once again displayed its arrogance and prejudice against a country that has made the most remarkable economic and social progress in the past three decades. The Nobel Prize has been generally perceived as a prestigious award in China, but many Chinese feel the peace prize is loaded with Western ideology [...] The award however makes it clearer that it is difficult for China to win applause from the West during China's development, and China needs to be more determined and confident in choosing its own development path, which is different from Western approach. ${ }^{3}$

Un tel discours est, on le sait, la résultante d'un double changement de paradigme dans la politique officielle chinoise où une certaine ouverture économique au capitalisme mondialisé va de pair avec une certaine fermeture politique vis-à-vis du libéralisme social :

Twice in the period of a little over two decades, party-stake leaders in China intervened deliberately to change the basic terms of the prevailing local political and cultural discourse in order to protect and enhance the ruling coalition's own legitimacy, and to better secure the official cultural hegemony. The first time just a little before the opening of the 1980', they shifted the main discursive motifs firmly away from the old Maoist maxims of 'revolution' and 'class struggle', on instead to the ideals of 'modernization'. The second time at the onset of the 1990's (though they did not by any means abandon all talk of 'modernizing' as such), the 
party-state shifted their broad discursive emphasis to themes of cultural nationalism, Chineseness, and the recovery of the glory of the great Chinese tradition. 4

Le discours du Global Times est le produit de cette double stratégie de modernisation économique et de nationalisme culturel qui se rejoignent dans la critique de la démocratie comme valeur étrangère, inadaptée à la Chine :

Since the June 1989 incident, in the view of the public, to talk about Enlightenment and democratization has become passé. The assumption is that one cannot still believe in these ideals when they so obviously do not work. On a pragmatic level it clearly means that, whatever it is that Chinese intellectuals do, they are doing it in the context of a discourse that says "democracy does not work or fit in China. ${ }^{5}$

Non pas que la démocratie soit rejetée comme concept, mais plutôt que sa définition officielle se confonde avec sa négation pratique $:^{6}$

The regime leadership has consistently held the orthodox view that democracy is based on the unity of interests both among the people and between the people and the Communist party, and that the party rules on behalf of the unified interests of the people. ${ }^{7}$

Définir la démocratie comme le pouvoir du «peuple » identifié aux représentants du Parti, c'est confondre la sphère sociale et politique en occultant le fait que tous les membres de la société ne disposent à l'évidence pas du même pouvoir dans cette société et sur ce parti, et empêcher la divergence de vues puisque la conformité de vue entre la société et le pouvoir est supposée être au fondement de la démocratie. Toute définition opposée à cette conception de la « démocratie » comme « dictature du prolétariat » et qui proposerait de définir la démocratie non pas comme protection et guidance du peuple par le pouvoir mais comme responsabilité du pouvoir et de l'Etat devant le peuple est caractérisée par le discours officiel comme contraire à l'intérêt vital du pays et étrangère à la culture du monde chinois. ${ }^{8}$

Notre propos dans cet article sera de proposer une archéologie philosophique de cette construction discursive pour montrer comment l'origine du discours officiel en Chine affirmant, contre « l'idéologie occidentale » responsable de l'attribution du prix Nobel à un « criminel » d'Etat, que la « Démocratie » ou les « Droits de l'Homme » sont des modèles étrangers, se trouve, par un paradoxe dont il faudra mesurer la portée par certaines de ces conséquences politiques (Taiwan...), en Occident même. Un tel discours utilise en effet, plus ou moins inconsciemment et stratégiquement, tout un ensemble rhétorique, argumentatif et catégorial qui non seulement s'est élaboré en Occident mais plus encore, dans un cadre eurocentriste, contre la Chine même. C'est donc aussi d'une archéologie de notre propre discours qu'il s'agit, d'une analyse critique de nos convictions eurocentristes et de notre foi éculée en l'existence d'une grande opposition Chine/Occident divisant l'esprit humain en deux moitiés antinomiques : "l'Occidentale », rationaliste et libre, et « l'Asiatique », poétique et « serve ».

\section{Récapitulatif d'un renversement européen des perspectives sur la}




\title{
Chine
}

Comme on le sait l'histoire des relations suivies, directes et attestées entre l'Europe et la Chine commencent véritablement au seizième siècle avec l'arrivée des missions jésuites et les succès complexes, relatifs, ambigus de Michele Ruggieri (1543-1607) et Matteo Ricci (1552-1610) à la cour de Wan Li (1563-1620).

8 De ces premières relations, il est commun de noter qu'elles furent fondées sur une incompréhension mutuelle : René Etiemble ironisant sur le fait qu'un confucéen croyant Dieu ne pouvait être qu'un... " Chrétien humaniste » ;9 Jacques Gernet soulignant le caractère « irréconciliable » du Ciel chinois et du Dieu chrétien ; ${ }^{10}$ Walter Mignolo rappelant le rejet, par ceux que Jean Lévi appelait les " fonctionnaires de la bureaucratie céleste », ${ }^{11}$ ou plutôt leurs héritiers à la fin de la dynastie Ming, de la vision du géographique du monde telle que la présentait le planisphère de Mercator (1562). ${ }^{12}$

Cependant, sur le plan méthodologique, nous voudrions déjà noter une chose : interpréter ces points de frictions et ces malentendus comme l'illustration d'une antinomie entre « la » Chine et « l'Europe » en général, c'est peut-être prendre un peu trop rapidement la partie pour le tout. Comme le note Peter C. Perdue :

\begin{abstract}
Westerners who brought the scientific revolution to China, filtering Western advances through religious screens. Jesuits, under orders from the Pope, concealed the prominence of the heliocentric model of the planetary system, instead promoting the compromise of Tycho Brahe, which still put the earth at the center, but placed the other planets orbiting the sun. Chinese interested in modern astronomy thus only received a partial view of the new science. ${ }^{13}$
\end{abstract}

Autrement dit, « l'Europe » ou « la culture européenne » que les Jésuites représentèrent auprès des lettrés de la cour impériale :

1. non seulement n'est pas toute l'Europe mais la partie de la tradition culturelle européenne qui a été élaborée par la tradition scolastique finissante, c'est-à-dire une retraduction partielle et partiale de la philosophie d'Aristote et de Platon pour qu'elle épouse les canons du christianisme officiel ;

2. mais plus encore n'est déjà plus tout à fait l'Europe, éclatée dans sa chrétienté par le schisme protestant, et plus encore débordée par la nouvelle pensée renaissante (retour au naturalisme atomiste antique source probable des futures révolutions scientifiques). ${ }^{14}$

Il est donc important de ne pas assimiler « l'Occident » avec la représentation «scolastique » orthodoxe que les Jésuites donnèrent de la « culture Européenne ».

14 De même assimiler « la Chine » avec le discours des administrateurs confucéens de la cour impériale consisterait au fond à accepter et entériner la version officielle orthodoxe (très prégnante de nos jours ${ }^{15}$ ) d'une culture chinoise essentiellement confucéenne d'où le taoïsme comme le bouddhisme seraient mystérieusement absents. ${ }^{16}$

15 Le deuxième moment des relations entre l'Europe et la Chine s'ouvre à l'époque des Lumières. C'est généralement une image extrêmement positive de la Chine que donnent les observateurs français (et européens) des dix-septième et dix-huitième siècles comme Le Comte dans ses Nouveaux mémoires sur l'état prése `t de la Chi`e (1696), J. B. du Halde dans sa Descriptio` de 
l'Empire de la Chi ee (1735). Voltaire dans son Dictio “aire philosophique comme dans son Essai sur les mours et l'esprit des 'atio `s n'a de cesse d'évoquer l'antiquité, la primauté et la profonde rationalité juridique et politique de la Chine : « une nation qui était toute policée quand nous n'étions que des sauvages. ${ }^{17}$ Dans le même esprit, Leibniz notait :

Ce serait une grande imprudence et présomption à nous autres nouveaux venus auprès d'eux, et sortis à peine de la Barbarie de vouloir condamner une doctrine si ancienne, parce qu'elle ne parait point s'accorder d'abord avec nos notions scholastiques ordinaires. ${ }^{18}$

Cependant, le terme « Chine » désigne alors moins un territoire aux contours géographiques et historiques déterminés que la mise en forme discursive d'un topos stratégique où il est moins question de la Chine que du rapport de l'Europe à elle-même.

Soit par exemple le discours relativement critique de Montesquieu sur la Chine dans l'Esprit des Lois: " La Chine est donc un État despotique, dont le principe est la crainte. ${ }^{19} \mathrm{Il}$ serait réducteur de qualifier ce discours d'antichinois : non pas tant parce que l'on peut trouver chez Montesquieu, dans ses notes $^{20}$, des propos plus nuancés, par exemple dans son appréciation de l'usage social des rites ${ }^{21}$, mais surtout parce que le but était moins la critique de la Chine comme telle que celle du despotisme dans le système politique en France. ${ }^{22}$ C'est contre les Jésuites, quoiqu'à partir de leurs notes sur la Chine, que les philosophes des Lumières, de Robert Challes ${ }^{23}$ à Voltaire, vont se référer à la culture chinoise pour montrer qu'une civilisation riche et raffinée peut être construite hors du christianisme et de la religion sur les bases d'une morale raisonnable incarnée, selon eux, par le Confucianisme. ${ }^{24}$

Enfin, troisième moment, le dix-neuvième siècle qui voit l'essor d'un discours eurocentriste et « sinophobe » duquel, c'est notre hypothèse, non seulement le discours européen sinophile mais aussi bien le discours chinois « europhobe » sont complètement tributaires.

Pour illustrer ceci, la philosophie de l'histoire de Hegel est exemplaire. Hegel, dans le cadre d'une évolution historique mondiale de l'Esprit des peuples où l'évolution collective de l'humanité est identifiée à l'évolution individuelle d'une personne de l'enfance à l'âge adulte ${ }^{25}$, identifie la Chine à l'enfance de la pensée. $^{26}$

20 Dès lors, ce qui va caractériser le peuple Chinois, comme sujet historique aux potentialités culturelles déterminées, c'est une aliénation à la pensée abstraite et à l'autonomie morale ${ }^{27}$ : infantilité spirituelle traduite par une incapacité culturelle à produire de la philosophie ${ }^{28}$, à élaborer une science valable et à construire un système politique autre que celui du despotisme paternaliste. ${ }^{29}$

Hegel par là jette les bases du cadre des « recherches sur la Chine » qui, jusqu'à peu, vont avoir pour but moins de remettre en cause ces idées que de les confirmer, en en cherchant les fondements soit 1. au niveau linguistique, soit 2. au niveau civilisationnel.

1. Si, de Humboldt ${ }^{30}$ à Heidegger ${ }^{31}$, le Grec et l'Allemand, voire pour Marcel Granet le français ${ }^{32}$, sont naturellement aptes à l'expression structurée d'une pensée abstraite et ontologique, le chinois lui en est structurellement dépourvu (thèse du mobilisme linguistico-métaphysique). C'est dans cette première perspective que se placerait une sinologie à la François Jullien, lequel n'aura eu de cesse, dans la lignée d'Heidegger, d'opposer l'ontologie métaphysique occidentale au mobilisme immanent chinois (et ce malgré le fait que la 
première ligne du texte le plus fameux de la philosophie chinoise affirme, opposant la fluidité du langage à la constance du principe, que « le Dao du dicible n'est pas le Dao du constant 》 道可道非常道). ${ }^{33}$

2. Quant au retard économique ${ }^{34}$ et technologico-scientifique ${ }^{35}$ du début de vingtième siècle, il fut compris comme la conséquence d'une morale confucéenne conservatrice : c'est la thèse de l'immobilisme culturel de la Chine dont découlerait l'impossibilité $i$ 'tri `sèque de penser la liberté sous la forme de l'Etat de droit et de la Démocratie. ${ }^{36}$ C'est dans cette seconde perspective que se placerait une sinologie à la Jean François Billeter, pour qui le despotisme oriental expliquerait à bien des égards les différentes formes de la vie intellectuelle en Chine - alors même que comme le disait Gernet, « l'idée d'une 'idéologie dominante' qui aurait été la marque d'un système politique immuable est parfaitement absurde ».37

\title{
Qu'est-ce qui s'est passé ? Archéologie du concept de Culture
}

Afin de tenter une analyse critique de cette double image de la Chine, nous voudrions essayer de mettre en cause son fondement même et tenter d'expliquer ainsi comment a pu s'opérer ce passage, souvent remarqué, de la sinophilie du dix-huitième siècle à la sinophobie du dix-neuvième siècle. ${ }^{38}$ Nous proposerons à cette fin une rapide archéologie de l'émergence même du concept de culture et de sa constitution au début du dix-neuvième siècle.

De fait, autant dans le Dictio “aire de Furetière publié en 1690 que dans l'E 'cyclopédie de Diderot et d'Alembert publié entre 1751 et 1772 , le terme " culture », si central dans les sciences humaines aujourd'hui, est absent. Il s'agit bien d'une invention récente.

En effet, promouvoir comme cadre d'identité sociale et politique la notion nouvelle de culture, au sens de " coutumes » d'un " peuple », impliquait une critique radicale de l'idéal rationaliste des Lumières. ${ }^{39}$ C'est pourquoi l'affirmation d'une " spécificité culturelle des peuples » est concomitante de l'émergence du nationalisme politique, d'abord en Allemagne et en Prusse $^{40}$, défendant l'identité spirituelle des peuples nationaux dans une opposition romantique et conservatrice à l'universalisme des Lumières ${ }^{41}$ et à la Révolution Française. ${ }^{42}$

27 De fait, plus précisément, la notion de culture s'invente dans le creuset des théories de la Volkgeist (notamment chez Humboldt à travers l'idée de déterminisme linguistique) $: 43$

\begin{abstract}
The shift in emphasis from "culture" as cultivation to culture as the basic assumptions and guiding aspirations of an entire collectivity - a whole people, a folk, a nation - probably occurred only in the nineteenth century, under the prompting of an intensifying nationalism. Then each people, with its characteristics culture, came to be understood as possessing a mode of perceiving and conceptualizing the world all of its own. 44
\end{abstract}

28 La notion " d'esprit des peuples » n'est en elle-même pas raciste au sens biologique ${ }^{45}$ mais plutôt xénophobe au sens culturel. ${ }^{46}$ Quoiqu'il en soit, en déterminant un groupe ethnique par des caractères spécifiques et homogènes, 
elle est en tout cas fondamentalement déterministe et conservatrice. ${ }^{47} \mathrm{La}$ co ‘ceptio` idéologique de la culture comme Volkgeist esse`tialise, homogé 'éise et isole les rapports culturels. Elle défi it la culture comme $u$ ' bloc homogè e (sa`s différe `ce `i diverge`ce i`ter`es) et éter el (sa`s co `tact `i méla`ge exter `es).

Or cette définition idéologique de la culture a largement contaminé la définition scientifique qui, au même moment, à la fin du dix-huitième siècle, se met en place avec la constitution de l'ethnologie comme pratique scientifique. C'est pourquoi il est vital de distinguer les deux.

On attribue parfois à Adam Franz Kollár, un historien slovaque, la paternité de la définition moderne de la notion de culture et celle corrélative de l'ethnologie qu'il définit comme :

\begin{abstract}
the science of peoples and nations, or, that study of learned men in which they inquire into the origins, languages, customs and institutes of various nations, and finally into the fatherland and ancient seats, in order to be able better to judge the nations and peoples in their own times. ${ }^{48}$
\end{abstract}

31 Ce qu'il y a de nouveau dans cette définition, c'est que le terme " culture » définit un ensemble de différe 'ces autour desquelles un groupe humain se construit (et non pas l'identité d'une civilisation opposée aux autres barbares). Une culture pourrait être définie comme le double produit d'une fonction auto-référentielle historique d'identification (affirmation de soi répétée dans le temps) et d'une fonction dialectique géopolitique de distinction (distance à l'autre formée dans l'espace) par laquelle un groupe humain se raconte (fabulation) en mettant en avant un ensemble de « traits » moraux et pratiques dont il reconnaît ou non (enjeu politique) à ses membres, différentiellement, la possession. ${ }^{49}$

\title{
Conséquences : Culture, Histoire et Démocratie
}

Tout ceci a d'importantes conséquences pour notre problème.

33 1. Première conséquence, la définition ethnologique, scientifique de culture, d'invention très récente en Europe, n'a pas de correspondant originel en Chine :

The term 'wenhua' does not translate [in China's minority policy] as 'culture' in the way it is used in Western social science. It is not a neutral term referring to economic strategies, social organization, ideology, values, and behaviors learned by people as members of a society. Rather, it is judgmental: one can speak in Chinese of people having a 'low' or a 'high' level of culture, and most of the national minorities are said to have a low or backward level of culture, or in a few cases to have no culture at all.50

Cette définition chinoise de la culture pourrait se comparer avec la conception grecque antique du terme culture dont Isocrate se faisait l'écho : 


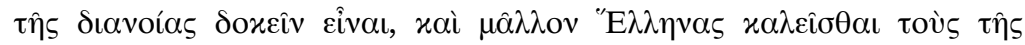

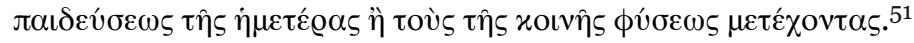

De même que, selon Isocrate, la culture est ce qui a fait la supériorité du peuple grec sur tous les autres mais qu'il est possible d'acquérir, non par le sang, mais par acculturation, de même, selon Ebrey:

Confucius and his followers over the centuries saw [Han] Chinese culture as superior to any other culture; they also saw as something outsiders could acquire..$^{2}$

En Chine ancienne comme en Grèce antique, le terme Culture sert de marqueur pour distinguer le Civilisé du Barbare : la possession de la forme culturelle du groupe dominant est non seulement la condition sine qua non de toute socialisation, mais elle est assimilée peu ou prou à une forme d'humanisation (du point de vue " confucéen », l'humanité rén 仁 de l'homme rén 人 s'acquiert par sa pratique lî 禮 de la vertu ỳ̀ 義 qui elle-même signifie à la fois respect des coutumes et connaissance de la langue/culture). Dans un tel cadre, la différence culturelle est impensable puisqu'il n'y a que deux domaines antagonistes : la Culture (Civilisation) et l'inculture (barbarie). Or tout l'enjeu consiste précisément à penser la différence (culturelle) autrement que sous la forme de l'opposition.

2. Deuxième conséquence, cette différence dans la conception du terme culture serait moins culturelle (on la retrouverait en Grèce) qu'épistémique, historique et relativement datable.

De fait, la relative convergence de la définition antique de la culture en Europe et en Chine fait écho à une autre possible convergence : celle, politique, existant entre Empire Romain et Empire Chinois pendant pratiquement un millénaire :

Two thousand years ago, perhaps half of the entire human species had come under the control of just two powers, the Roman and Han empires at opposite ends of the Eurasia. Both entities were broadly similar in terms of size. Both of them were run by god-like emperors residing in the largest cities the world had seen so far; both states were made up of some 1,500 to 2,000 administrative districts and, at least at times, employed hundreds of thousands of soldiers. Both states laid claim to ruling the whole world, 'rbis terrarum and tianxia, while both encountered similar competition for surplus between central government and local elites, similar pressures generated by secondary state formation beyond their frontiers and subsequent 'barbarian' infiltration, and both of them even ended in similar ways. 53

Ce n'est qu'à partir du sixième siècle, toujours selon Walter Scheidel, que les divergences s'accentuent avec la décomposition de l'Empire Romain menant à la constitution des Etats-Nations. ${ }^{54}$ Or cette formation progressive des Etats Nations, à partir de la Renaissance, en Europe, aura entrainé, c'est notre hypothèse, une compétition technologique, industrielle et militaire entre puissances régionales dont la conséquence externe furent le colonialisme, la conséquence interne le progrès scientifique et, à l'interface, le capitalisme " militaro-industriel »- toutes choses dont, à rebours, l'unification impériale de la Chine n'aura pas pu permettre, assez tôt pour ne pas en devenir la victime, l'émergence. ${ }^{55}$

3. Troisième conséquence, on peut mieux saisir dès lors l'erreur méthodologique du discours eurocentriste. Comme le dit James Blaut dans sa 
critique de Weber, elle consiste à avoir assimilé ce qui était le fruit d'une divergence historique contingente, progressive et réversible à une différence culturelle déterminante, éternelle et essentielle (supposer une grande divergence Chine/Occident fondée sur la géographie masque les nombreuses analogies historiques entre Chine et Grèce au cours du temps et empêche la datation précise du moment de l'essor des divergences). ${ }^{56}$

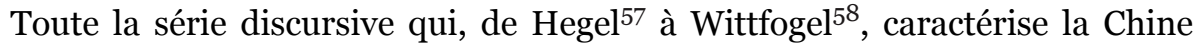
par l'immobilisme liberticide est une construction idéologique visant :

1. à édifier le mythe d'une union naturelle entre l'Occident et la liberté ;

2. à masquer la pratique réelle du colonialisme européen à la même époque en érigeant le contre-modèle du « despotisme asiatique ».

Il est évident pourtant qu'une telle mythologie culturelle ne résiste pas aux faits ${ }^{59}$, à l'évidence historique que la Liberté politique sous sa forme constitutionnelle ou " démocratique ", loin d'être consubstantielle à « l'Occident », lui a été le plus souvent totalement étrangère :

Throughout most of the Western history, democracy far from being the establishment, was virtually unknown. With an exception of Western Athens, where democracy generally prevailed from roughly, 450 to 322 $\mathrm{BC}$, no example of a democratic regime appeared during the subsequent two thousand years. Even in the constitution of our [American] Founders democracy was only one element and by no means the most valued. Only in twentieth century were the political regimes that were democratic, when judged by formal criteria as such as universal franchise for adult citizens, legal rights to which all the citizens are equally entitled, a free press and political parties and comprehensive public educational systems. ${ }^{60}$

4. Quatrième conséquence, on comprend dès lors également pourquoi, plutôt que de mettre en question l'identité supposée de l'Occident et de la Liberté (dont la forme politique serait la Démocratie comme forme de participation plurielle de la société à la politique interne de l'Etat impliquant la mise en demeure possible de l'Etat par les membres de la société), le discours officiel en Chine préfère reprendre à son compte le mythe eurocentriste et sinophobe du « despotisme asiatique » fondant l'absence de liberté politique en Chine sur une donnée culturelle immémoriale et intangible. Par un étrange paradoxe, la réappropriation du mythe eurocentriste " sinophobe » constitue le pivot central du discours sinocentriste « europhobe ».

5. Cinquième conséquence, les limites du discours européen sinophile apparaissent de façon plus claire. Quand François Jullien affirme :

La notion de liberté est-elle aussi universelle qu'on le prétend aujourd'hui en Occident, en revendiquant le modèle de la démocratie ? On découvre en Chine une autre pensée politique qui est passée à côté de la Liberté. ${ }^{61}$

Cela pose au moins deux vrais problèmes :

1. Comment affirmer une telle proposition sans faire l'archéologie de son propre discours qui nous permettrait d'en ressaisir les fondements eurocentristes ? Ce n'est pas vouloir la démocratie en Chine qui est eurocentriste mais affirmer, au contraire, qu'elle ne saurait y survenir.

2. Comment affirmer une telle proposition sans avoir la conscience de sa résonnance contemporaine ? En Chine même, la Démocratie étant devenue un enjeu de pouvoir dans l'appareil politique et de contrôle de l'organisation 
sociale, une telle affirmation constitue de fait la meilleure justification de la frange la plus conservatrice du Parti et de la société pour bloquer tout progrès :

En octobre 2004, l'Assemblée populaire nationale a décidé d'allonger de trois à cinq ans le mandat des gouvernements de bourg et de canton [...] Ces élections représentaient alors, pour beaucoup de réformateurs, une nouvelle occasion d'approfondir les expérimentations. À leur grande déception, M. Sheng Huaren, vice-président du Comité permanent de l'Assemblée populaire nationale, a cependant déclaré en août 2006 que l'élection directe des chefs de gouvernements cantonaux était illégale et a attiré l'attention sur le fait que des Occidentaux mal intentionnés invoquaient le principe des droits de l'homme et de la démocratie pour nuire à la Chine. ${ }^{62}$

\section{Etude de cas : Taiwan ou le grain de sable du réel dans la mécanique idéologique}

Nous évoquerons enfin le cas de Taiwan pour montrer, à travers un cas concret, combien un discours sans fondement ni historique ni philosophique peut être pour le pouvoir éminemment utile. Le discours officiel sur Taiwan est en effet confronté à un vrai paradoxe :

1. On a vu que la "Démocratie » (système de représentation du "peuple » par des élus choisis sur la base de leur offre politique différentiée et soumission du gouvernement au pouvoir au respect des lois fondamentales de l'Etat auquel chaque citoyen peut faire appel de façon légitime) était dite incompatible avec les valeurs culturelles spécifiquement chinoises.

2. On sait d'autre part que la réintégration de Taiwan dans la Chine continentale est considérée par certains intellectuels chinois comme une priorité nationale vitale ${ }^{63}$ et qu'une des raisons avancées pour justifier cette réintégration est celle d'une supposée identité culturelle (« confucéenne ») ou ethnique (Han). ${ }^{64}$

3. On sait enfin que Taiwan s'est démocratisée ${ }^{65}$ et forme une démocratie au fonctionnement indépendant quoique perpétuellement sous tension. ${ }^{66}$

Il y a donc une contradiction flagrante entre les présuppositions 1. et 2. la réalité 3 . :

The existence of a democratic Taiwan provides practical demonstration that this type of political regime can be made work in a Chinese setting a demonstration with far-reaching implications for China, Hong-Kong, Singapore, and for those who theorize about supposed 'Asian-values' or the 'clash of civilizations'...67

En effet, de deux choses l'une (chacune étant impossible dans la logique officielle) :

1. soit on affirme que la culture chinoise est incompatible avec la démocratie et dès lors Taiwan s'étant démocratisée ne ferait plus tout à fait partie de « la » culture chinoise et n'aurait pas à faire partie de la Chine - ou bien ne pourra en faire partie que par une "dé-démocratisation » impliquée ${ }^{68}$ ou forcée (c'està-dire la guerre) $;$;9

2. soit on affirme que Taiwan doit être intégrée à la Chine sur la base d'une 
(supposée) «identité » culturelle et ethnique et donc, dès lors, si Taiwan a réussi à se démocratiser, en tant que la culture de Taiwan et celle de la Chine continentale seraient « similaires », cela impliquerait que la démocratie n'est pas contraire à « la » culture chinoise et que la Démocratie est possible en Chine continentale également. Or, dernier paradoxe et cercle vicieux, ce qui s'oppose à une telle conséquence, c'est justement que, pour le pouvoir central, une telle démocratisation pourrait entraîner des choix d'indépendance, notamment de Taiwan :

The Chinese leadership refuses to adopt a democratic approach, that would allow the people of Tibet and Taiwan to choose for themselves to be part of, or separate from, the Chinese nation. Chinese state nationalists firmly oppose democratization, which they see as a threat to China's control of the territories and hence to national unity. ${ }^{70}$

Autrement dit, le discours sur la spécificité culturelle chinoise dont le pouvoir s'empare pour s'opposer, officiellement, au type d'activité politique conduite par le prix Nobel de la paix 2010 et à toute démocratisation du régime en place a une valeur stratégique de contrôle tout autant populationnelle (contre l'autonomie collective) que territoriale (contre la " décolonisation » intérieure). ${ }^{71}$ Mais le paradoxe et l'ironie est qu'en affirmant, pour des exigences de contrôle, que les valeurs démocratiques sont étrangères à la Chine, le discours officiel conforte, pour appuyer son propre pouvoir et afficher sa résistance aux pressions étrangères, le schéma de penser le plus « occidental» (et antichinois) qui soit ${ }^{72}$ : ce qui, en termes de soft-power est, de façon évidente, durablement contre-productif et ne fait que renforcer et souligner l'(inacceptable) indépendance de fait d'une Taiwan démocratisée. ${ }^{73}$

\section{Conclusion : penser la différence sans l'opposition}

Notre propos dans cet article était d'étudier les rapports entre culture et politique sous l'angle double de la Démocratie comme principe politique et de la manière dont les intellectuels européens ont pensé le rapport à la Chine. Un des moments pivots de l'histoire des relations culturelles entre la Chine et l'Europe est la construction, notamment en Allemagne, au début du dix-neuvième siècle de la notion de Volkgeist. La notion de Volkgeist a servi plus ou moins inconsciemment jusqu'à nos jours de cadre de référence à la pratique comparatiste. Son présupposé métaphysique le plus fort est d'interpréter la Différence en termes d'Opposition et de briser l'Universalité transcendantale de la subjectivité humaine en une série d'isolats culturels antinomiques entre eux.

Penser l'Autre non comme Opposé mais comme Différent, non comme séparé par la Géographie mais réuni par l'Histoire, non comme une forme figée et isolée mais une force évolutive et interactive, permettrait de s'affranchir des présupposés de la notion de Volkgeist sans retomber dans les travers d'un universalisme homogénéisant. Or une fois levée l'hypothèque de la Volkgeist comme cadre de référence des phénomènes culturels, un vaste chantier s'ouvre au sein duquel l'ensemble des oppositions établies entre la Chine et l'Europe pourraient être à revoir : opposition supposée entre une métaphysique 
ontologique grecque et une pragmatique du devenir chinoise ${ }^{74}$, opposition supposée entre un droit civil européen et un code pénal asiatique ${ }^{75}$, opposition supposée entre une science abstraite occidentale et un savoir pratique chinois... ${ }^{76} \mathrm{Il}$ serait donc temps que le discours sinologique sorte du débat figé entre penser la Chine sous la figure de l'Autre (Jullien) ou penser la Chine sous la figure de l'Universel (Billeter) et puisse intégrer dans sa méthodologie la manière dont la philosophie française contemporaine (Deleuze, Derrida, Foucault...) a su penser la Différence comme ni Autre ni Même (mais Multiplicité interne).

A l'intérieur de ce vaste chantier, la problématique des rapports entre Liberté et Démocratie a une importance stratégique pour la Chine d'aujourd'hui. De ce point de vue, pour revenir au problème plus spécifique de la Démocratie en Chine, on peut se demander ce qui serait le plus douloureux pour le pouvoir chinois dans la transition en droit possible et sans doute nécessaire (quoiqu'en fait indéfiniment ajournable) de la société civile vers la « démocratie » ou l'ouverture des libertés publiques (expression, contestation, recours) : prendre le risque d'une autonomisation des régions limitrophes et d'un renversement des élites politiques du Parti ou bien plutôt abandonner un discours culturaliste si fédérateur et si fondé dans l'imaginaire d'une grande séparation Chine/Occident?

\section{Notes}

1 Pinyin: Zhōngguó bìxū lìjí kāishǐ shíxíng xiàliè liăng fāngmiàn de mínzhŭ găigé. dì yĩfāngmiàn, jiāng zhèngzhì zhìdù shàng Guómíndăng yĩ dăngpài yĩ jiēeji de făndòng dúcái zhèngtǐ, găi biànwéi gè dăngpài gè jiēejí hézuò de mínzhŭ zhèngtǐ. Zhè fāngmiàn, yìngcóng găibiàn guómín dàhuì de xuănjŭ hé zhà ‘ji shàng wéifăn mínzhǔ de bànfă, shíxíng mínzhŭ de xuănjŭ hé bă zhèng dàhuì de zìyóu kāihuì zuò qŭ, zhídà ‘ zhiding zhēnzhèng de mínzhŭ xiànfă, zhà ’jí zhēnzhèng de mínzhǔ guóhuì, xuănjŭ zhēnzhèng de mínzhǔ zhèngfŭ, zhíxíng zhēnzhèng de mínzhǔ zhèngcè wéizȟ̌. Mao Tse-Tung, "The Tasks of the Chinese Communist Party in the Period of Resistance to Japan", May 3, 1937 in Selected W`rks `f Ma` Tse-tung, Foreign Languages Press Peking 1967, Vol. I, pp. 267-8: "China must at once start democratic changes in the-two following respects. First, in the matter of the political system, the reactionary Kuomintang dictatorship of one party and one class must be changed into a democratic government based on the co-operation of all parties and all classes. In this respect, a start should be made by changing the undemocratic procedures for electing and convening the national assembly, and by holding democratic elections to the assembly and ensuring freedom in the conduct of its meetings, after which it will be necessary to go on to framing and adopting a truly democratic constitution, convening a truly democratic parliament, and electing a genuinely democratic government that will carry out genuinely democratic policies."

2 Jingsheng Wei,San Huang, La cinquième m`dernisati`n et autres écrits du «Printemps de Pékin », Paris, Christian Bourgeois, 1997.

3 “2010 Nobel Peace Prize a disgrace”, Gl `bal Times, October 09, 2010

4 Vivienne Shue, "Global imaging, the state's quest for hegemony, and the pursuit of phantom freedom in China”, Gl'balizati 'n and dem 'cratizati $n$ in Asia: the c 'nstructi`n ‘fidentity, Catarina Kinnvall and Kristina Jönsonn, Routledge 2002, p. 21, p. 215 .

5 Ben Xu, Disenchanted dem `cracy: Chinese cultural criticism after 1989, University of Michigan Press, 1999, p. 3-4.

6 Zhang Lun, « L'intellectuel, le pouvoir et l'idée de démocratie après Mao; discours et pratiques », La Chine et la Dém 'cratie, Mireille Delmas-Marty et Pierre-Etienne Will (Dir.)., Fayard, 2007, p. 517-547 : « Les libertés et les droits individuels - la liberté d'expression, de publication, d'association, etc. - ainsi que la plupart des principes 
modernes d'organisation politique présent dans les Constitutions des pays démocratiques existent également dans les versions successives de la Constitution de la République populaire de Chine, depuis la première, celle de 1954. Pourtant l'histoire montre à l'envi à quel point ces droits et ces principes ont été privés de toute application sérieuse. »

7 Yijiang Ding, Chinese Dem `cracy After Tiananmen, Vancouver, UBC Press, 2001, p. 16.

8 Pierre-Etienne Will, « Le contrôle de l'excès de pouvoir sous la dynastie des Tang », La Chine et la Dém `cratie, Mireille Delmas-Marty et Pierre-Etienne Will (Dir.)., Fayard, 2007, p. 111-156 : en Chine, " [l'Etat] n'existe pas en tant qu'institution juridique susceptible d'être mise en cause devant une instance indépendante et dont on pourrait exiger une réparation. Sur ce plan, l'Etat et les institutions qui le composent se confondent avec les personnes. " Susan L. Shirk, "The Chinese Political System and the Political Strategy of Economic Reform", in Kenneth G. Lieberthal and David M. Lampton (Dir.), Bureaucracy, $P$ `litics and Decisi $n$ making in $P$ `st-Ma`China, University of California Press, 1992, pp. 59-91: "The crucial difference between communist and democratic systems is the political accountability of the principals".

9 René Etiemble, L'Eur `pe Chin `ise, Paris, Gallimard, 1988, vol. 1, p. 187.

10 Jacques Gernet, La Chine et le christianisme, Paris, Gallimard, 1982, p. 198.

11 Jean Lévi, « Les fonctionnaires et le divin : luttes de pouvoirs entre divinités et administrateurs dans les contes des six Dynasties et des Tang ", Cahiers d'Extrême Asie, 1986, vol. 2, $\mathrm{n}^{\circ} 2$, pp. 81-110 ; "Les fonctions religieuses de la bureaucratie céleste », L’H 'mme, $1987, \mathrm{n}^{\circ} 101$, pp. 35-57.

12 Walter Mignolo,The Darker Side ' $f$ the Renaissance: Literacy, Territ 'riality, and $C ` l$ nizati $n$, University of Michigan Press, 1995, pp. 224-225.

13 Peter C. Perdue, “Chinese Science: a Flexible Response to the West?", East Asian Science, Techn ` $` g y$ and $S$ `ciety: an Internati `nal J urnal (2007) 1:143-145.

14 David C. Lindberg, The beginnings 'f western science: the Eur 'pean scientific traditi $n$ in Phil `s `phical, Religi `us and Instituti `nal C`ntext, Prehistory to AD. 1450, University of Chicago Press, 2007, p. 365.

15 Voir par exemple : You Nuo, "Modern China needs some old thinking”, China Daily, July 31, 2006: "Confucianism is something very Chinese and irreplaceable in this society. It is not science, or anything from which an analytical model can be developed. However, it is the main part of this society's moral tradition, or how people tell right from wrong". Comme le dit ThomasBoutonnet, "Traitement moral de la question sociale dans la « société harmonieuse » de Hu Jintao »,Transtext(e)s Transcultures 跨 文本跨文化, 2009, $\mathrm{n}^{\circ} 5$ : “Cette “confucianisation" du discours politique permet d'installer cet ensemble d'énoncés dans une certaine "sinité" tout en lui offrant un cadre idéologique et moral très efficace et favorable au maintien de l'ordre établi. »

16 Franklin Perkins, Leibniz and China: a c `mmerce `f light, Cambridge, Cambridge University Press, 2004, p. 15: "The Jesuits missionaries associated primarily with the class of Confucian scholar officials, the ru or "literati," and these served as their main source on Chinese thought. They were thus introduced to an orthodox form of what has come to be called "Neo-Confucianism,' while remaining ignorant and dismissive of Buddhism and Daoism."

17 Voltaire, Essai sur les mœurs et l'esprit des nati $n s$ et sur les principaux faits de l'hist 'ire, depuis Charlemagne jusqu'à L 'uis XIII, Vol 1, Chap. I. - De la Chine, de son antiquité, de ses forces de ses lois, de ses usages et de ses sciences.

18 G. W. Leibniz, "Lettre sur la philosophie chinoise à Nicolas de Rémond », Zwei Briefe über das Binare Zahlensystem und die chinesische Phil `s `phie. Stuttgart, BelserPresse, 1968, p. 39-132. Albert Ribas, "Leibniz' discourse on the natural theology of the Chinese and the Leibniz-Clarke controversy”, Phil `s `phy East and West, January 2003.

19 Montesquieu, De l'Esprit des L `is, Première Partie, Livre VIII.

20 Ge `graphica, Masson, t. II, 1950, p. 923-963 ; Euvres C`mplètes, t. XVI, Oxford, Voltaire Foundation, C. Volpilhac-Auger eds.

21 C. Volpilhac-Auger, "On the proper use of the stick: TheSpirit ' $f$ Laws and the Chinese Empire”, in Rebecca Kingston, M`ntesquieu and his legacy, Albany, State University of New York Press, 2009, pp. 81-96. 
22 Simon Kow, The Idea 'f China in M`dern P`litical Th 'ught: Leibniz and $M$ 'ntesquieu, Canadian political association papers, 2005 : "The comparison of monarchy and despotism is arguably intended to be a critique of French absolutism, as scholars have noted. In this light, it was important to Montesquieu that China not be regarded as an example of enlightened despotism".

23 Robert Challes, J'urnal d'un v'yage fait aux Indes 'rientales, Rouen : Jean-Baptiste Machuel, 1721, vol. III, p. 150 : " A l'égard de leurs Casuistes, \& des Idolâtries des Chinois et des leurs, dans la Chine, ne pouvant démentir des Faits si graves, \& si bien prouvés, ils se sont mis sur le pied de vouloir les justifier. »

24 H. Nakagawa, " Les confucianistes, philosophes tolérants dans la pensée de Voltaire », Revue Internati `nale de Phil `s `phie, 1994, vol. 48, n¹87, pp. 39-53.Takeshi Koseki, "Diderot et le confucianisme : autour du termeJu-kiaode l'article ${ }^{*}$ Chinois (Philosophiedes) »,Recherches sur Dider 't et sur l'Encycl 'pédie, 1994, vol.16, nº 16, pp. 125-131.

25 Hegel, Phil `s `phie der Geschichte, Werke, vol. 12, pp. 72-73: „Dieser Geist eines Volkes ist ein bestimmter Geist [...] der bestimmte Volksgeist selbst nur ein Individuum ist im Gange der Weltgeschichte" Traduction : Cet esprit d'un peuple est un esprit déterminé. [...] qui doit être considéré comme seulement un Individu dans le cours de l'Histoire Universelle.

26 Hegel, V`rlesungen über die Phil `s `phie der Geschichte, Philipp Reclam Jun., 1997, p. 168: „Die Weltgeschichte geht von Osten nach Westen, denn Europa ist Schlechthin das Ende der Weltgeschichte, Asien der Anfang“. Traduction : L’Histoire Universelle va d'Est en Ouest ; l'Europe est en soi la fin de l'Histoire Universelle et l'Asie son commencement.

27 Georg F. W. Hegel, The phil `s `phy `f hist`ry, traduction J. Sibree, New York, Barnes \& Noble, 2004, p. 153: "This is the character of the Chinese people in its various aspects. Its distinguishing feature is that everything which belongs to Spirit unconstrained morality, in practice and theory, Heart, inward Religion, Science and Art properly so called - is alien to it”.

28 G.W.F. Hegel, V`rlesungen über die Geschichte der Phil`` ’hie, I, 18, Herausgegeben von Eva Moldenhauer und Markus Michel, Suhrkamp Verlag, Frankfurt am Main, 1971, 142f: „Aus seinen Originalwerken kann man das Urteil fällen, daß es für den Ruhm des Konfutse besser gewesen wäre, wenn sie nicht übersetzt worden wären“

29 Georg F. W. Hegel, The phil `s ’phy `f hist`ry, traduction J. Sibree, New York, Barnes \& Noble, 2004, p. 124, 137: "This paternal care on the part of the Emperor, and the spirit of his subjects - who like children do not advance beyond the ethical principle of the family circle, and can gain for themselves no independent and civil freedom makes the whole an empire, administration, and social code, which is at the same time moral and thoroughly prosaic - that is, a product of the Understanding without free Reason and Imagination".

30 Martin Bernal, Black Athena writes back: Martin Bernal resp 'nds $t$ ' his critics, David Chioni Moore ed., Duke University Press, 2001, pp. 119-20: "Humboldt, however, was convinced that "Sanscritic," that is to say Indo-European languages were qualitatively different from and superior to all others. Furthermore in an outline of the new discipline, later known as Altertumwissenschaft or "Classics", he declared that the excellence of Greek lay in its being uncontamined by foreign elements. Elsewhere, he maintained that Greek history and culture as a whole were categorically above that all others cultures and that 'from the Greeks we take something more than earthly - almost godlike'."

31 Heidegger, Intr `ducti ` $n$ à la métaphysique, cité et traduit par Derrida, De l'esprit Heidegger et la questi $n$, Galilée, p. 1987, p. 109 : " Le fait que la formation de la grammaire occidentale soit due à la réflexion grecque sur la langue grecque donne à ce processus toute sa signification. Car cette langue est avec l'allemande (au point de vue des possibilités du penser), à la fois la plus puissante de toutes et celle qui est la plus spirituelle. »

32 Marcel Granet, "Quelques particularités de la langue et de la pensée chinoise », Etudes s `ci 'l 'giques sur la Chine, P.U.F., 1990, pp. 99-155 : «Tandis qu’un Français, par exemple, possède, avec sa langue, un merveilleux instrument de discipline logique, mais doit peiner et s'ingénier s'il veut traduire un aspect particulier et concret du monde sensible, le Chinois parle au contraire un langage fait pour peindre et non pour classer, un langage fait pour évoquer les sensations les plus particulières et non pour définir et 
pour juger, un langage admirable pour un poète ou un historien, mais le plus mauvais qui soit pour soutenir une pensée claire et distincte, puisqu'il oblige les opérations qui nous semblent les plus nécessaires à l'esprit, à ne se faire que de façon latente et fugitive. »

33 Martin Heidegger, Schelling’s Treatise `n the Essence ‘f Human Freed `m, Ohio University Press, 1985, 146: "For the great beginning of Western philosophy, too, did not come out of nothing. Rather, it became great because it had to overcome its greatest opposite, the mythical in general and the Asiatic in particular, that is, it had to bring it to the jointure of a truth of Being, and was able to do this".

34 Max Weber, The Religi ` ` $f$ China, Hans H. Gerth traduction, New York Free Press, 1968, p. 104: "rational entrepreneurial capitalism, which in Occident found its specific locus in industry, has been handicapped not only by the lack of a formally guaranteed law, a rational administration and judiciary, and by the ramifications of a systems of prebends, but also, basically, by the lack of a particular mentality [...] In particular it had been handicapped by the attitude rooted in the Chinese 'ethos' and peculiar stratum of officials and aspirants to office."

35 Max Weber, „Vorbemerkung“, Religi`nss `zi`l`gie I, Institut für Pädagogik der Universität Potsdam, 1999: Nur im Okzident gibt es "Wissenschaft" in dem Entwicklungsstadium, welches wir heute als "gültig" anerkennen. Traduction: seulement en Occident la Science a atteint le degré de développement suffisant pour produire des résultats reconnus aujourd'hui comme valides.

36 Kjed Erik Brodsgaard, "Confucianism in mainland China today”, in N`rms and the State in China, Chun-Chieh Huang et Erik Zürcher ed., E.J. Brill, 1993, pp. 168-184: "Weber found that China could not develop a modern, rational capitalism from indigenous sources because of a host of factors [...] According to Weber the Chine Confucianists lacked "the central religiously determined and rational method of life which came from within and which was characteristic of the Classical Puritan."

37 Cité par Patrice Fava, «À propos de C`ntre Franç `is Jullien », Etudes Chin `ises, vol. 25, 2006, p. 173-198.

38 Michel Cartier, La Chine entre am `ur et haine, Actes du VIIIcolloque de sinologie de Chantilly, Desclée de Brouwer, 1998.

39 Eric R. Wolf, Envisi `ning p`wer: ide ` `gies `f d`minance and crisis, Berkeley: University of California Press, 1999, p. 22: "The issue of Reason against Custom and Tradition was raised by the protagonists of Enlightenment against their adversaries, the advocates of what Isaiah Berlin called the Counter-Enlightenment."

40 Harro Segeberg, "Germany”, Otto Dann, John Rowland Dinwiddy, ed., Nati 'nalism in the age 'f the French Rev 'luti 'n, Hambledon Press 1988, p. 137-157 (142, 144): "Given the originally cosmopolitan character of the Enlightenment, it is somewhat surprising that national awareness formed a continuous strand in the thinking of this newly middle-class culture [...] The theme of differences in national character, especially between French and Germany took on an increasing importance. This discussion subsequently played a major role in determining German attitudes to French revolution."

41 Matti Bunzl, "Frantz Boas and the Humboldtian tradition”, $V^{`}$ 'lkgeist as Meth 'd and Ethic, Essays 'n B `asian ethn `graphy and the German anthr 'p $l^{\prime}$ gical traditi $n$, George W. Stocking eds., University of Wisconsin Press, 1996, p. 17-78: "In opposition to the French Enlightenment, which based its universalism on the essential sameness of human beings as rational actors, Herder stressed the individual contribution of each cultural entity to humanity at large. And since individuality was the totality of its multitudinous elements, each Volk must be studied in its individuality."

42 Franz Dumont "Rhineland" in Otto Dann, John Rowland Dinwiddy, ed., Nati 'nalism in the age 'f the French Rev 'luti ' $n$, Hambledon Press 1988, p. 163: "the Germans were collectively characterized as open, upright, and God-fearing, while the French were portrayed as frivolous and unreliable [...] It was claimed that whereas the French had a natural penchant for revolution, the Germans are by nature averse to it. The trustiness (Biedersinn) of the Germans involved loyalty to the 'legitimate authorities', obedience to the 'laws from above', piety and the fear of God, respect for traditions and the achievements of the past, and an instinctive distrust of new-fangled foreign ideas". Si l'on remplace Allemands par Chinois, Français par Occidentaux et Révolution par Droits de l'Homme et Démocratie, on retrouvera la même structure argumentative dans les pamphlets nationalistes chinois. 
43 Matti Bunzl, "Frantz Boas and the Humboldtian tradition”, $V^{`}$ lkgeist as Meth `d and Ethic, Essays 'n B `asian ethn `graphy and the German anthr ' $p$ ' ${ }^{\prime}$ gical traditi $n$, George W. Stocking eds., University of Wisconsin Press, 1996, p. 17-78: "Humboldt believed that the national character of a people driven by its inner forces, or $V^{`} l k s g e i s t$, determined and was manifested in a variety of cultural aspects, including its customs and morals. Most important, however, Nati 'nalcharakter decisively affected the language of each individual "tribe ( $V^{`}$ lkerstämme), which was the direct product of its "spiritual peculiarity" (Geistegeseingenthümlichkeit): "Language is the external representation of the genius of peoples".

44 Eric R. Wolf, Envisi 'ning p `wer: ide`l'gies `f d’minance and crisis (Berkeley, University of California Press, 1999), p. 29.

45 Eric Storm, "Regionalism in History, 1890-1945: The Cultural Approach”, Eur 'pean Hist 'ry Quarterly, April 2003, Vol. 33, $\mathrm{n}^{\circ}$ 2, p. 251-267: "Nevertheless, racial doctrines were fundamentally based on a biological determinism, whereas the $V^{\prime}$ 'lksgeist theory presupposed that people interact with their environment. Whereas the physical milieu and historical traditions determine the character of a people, they are to a certain extent shaped and transformed by the people. Racial factors could play a role, but were never predominant."

46 Jan Noordegraaf, The Dutch Pendulum. Linguistics in the Netherlands 1740-1900, Münster: Nodus Publikationen 1996, pp. 86-98: "The V'lksgeist concept has haunted Dutch linguistics for quite some time. [...] According to this yard-stick the Afrikaans spoken by the Boers was a degenerate language, which had regressed to a primitive state due to long-standing contacts with savage peoples."

47 Hans Bernhard Schmid, Plural Acti 'n: Essays in Phil `s `phy and S 'cial Science, London: Springer, 2009, p. 182: "Even in the case of the pre-Nazi notion of V'lksgeist, the aim behind the concept is to conceive of social identity in terms of what people are instead of in terms of what they $d$ '. Even here, the concept is accompanied by more or less overt depreciation of both individual autonomy and collective democratic self-determination."

48 Han F. Vermeulen, Early Hist 'ry 'f Ethn 'graphy and Ethn 'l'gy in the German Enlightenment: Anthr 'p ' ' 'gical Disc 'urse in Eur `pe and Asia, 1710-1808, PhD thesis University of Leiden / Proefschrift Universiteit Leiden, 12 November 2008, Conclusion.

49 Frederik Barth, Ethnic gr 'ups and b 'undaries. The s `cial 'rganizati 'n 'f culture difference (Oslo: Universitetsforlaget, 1969).

50 Norma Diamond, "Ethnicity and the state: the Hua Miao of Southwest China", Ethnicity and the state, Judith Drick Toland eds., Transaction Publishers (1993) 2009, 55-78.

51 Isocrates, Panegyricus, § 50, Harvard University Press 1980, George Norlin trad: "And so far has our city distanced the rest of mankind in thought and in speech that her pupils have become the teachers of the rest of the world; and she has brought it about that the name Hellenes suggests no longer a race but an intelligence, and that the title Hellenes is applied rather to those who share our culture than to those who share a common blood."

52 Patricia Ebrey, "Surnames and Han Chinese Identity" in Melissa J. Brown eds., Neg 'tiating Ethnicities in China and Taiwan, Berkeley, CA: University of California Press, 1996, p. 20.

53 Walter Scheidel, "From the 'Great Convergence' to the 'First Great Divergence': Roman and Qin-Han state formation and its aftermath", Rome and China: comparative perspectives on ancient world empires, Walter Scheidel ed., Oxford University Press, 2009, p. 11.

54 Ibid "It was only from the late sixth century CE onward that the two trajectories of state formation began to diverge, slowly at first but more dramatically over time, between the cyclical restoration of a China-wide empire in the East and the decline of empire and central government in the West, followed by the slow creation of a polycentric state system that proved resistant to any attempts to impose hegemony, let alone unification, and ultimately evolved into the now-familiar cluster of modern nation states.”

55 David Held, Anthony G. McGrew, Gl `balizati`n the `ry: appr `aches and c 'ntr 'versies, Cambridge: Polity Press, 2007, p. 18-9: "At the congress of Vienna in 1815 Europe's great powers sought to manage their continental competition with the 
consequence that it was largely displaced into a struggle for global mastery. Capitalist industrialization reinforced this process since it required healthy supplies of raw materials and new markets while simultaneously revolutionizing the instruments, organization and lethality of warfare [...] Transformations in communication and transport technologies, from the steamship and railway to the telegraph, enhanced and accelerated the logistics of building, as well as the infrastructural capacity for ruling, empires of a global reach.”

56 James Morris Blaut,Eight Eur `centric hist 'rians, Guilford Press, 2000, p. 25-6: "European science, mathematics, and technology were in no way higher than Chinese and Indian science prior to early modern times. After the rise of Europe, and particularly after the industrial revolution, you can expect both a flowering science prior and an awesome increase in the scale and opulence of all other accomplishments - for instance, huge orchestras. But if comparisons are made for the period before 1492, when many of the world's civilizations were truly medieval, then Europe does not stand out. Not in science, not in art, no in law, not in the development of capitalism."

57 Georg F. W. Hegel, The phil `s `phy 'f hist `ry, traduction J. Sibree, New York: Barnes \& Noble, 2004, p. 129: "Early do we see China advancing to the condition in which it is found at this day; for as the contrast between objective existence and subjective freedom of movement in it, is still wanting, every change is excluded, and the fixedness of a character which recurs perpetually, takes the place of what we should call the truly historical."

58 Karl A. Wittfogel (1957), "Oriental Despotism: a comparative Study of Total Power”, The f'undati 'ns 'f bureaucracy in ec ' $n$ 'mic and s'cial th 'ught, vol. 1, Bill Jenkins, Edward C. Page, Edward Elgar Publishing, 2004, pp. 105-126: "In virtually all hydraulic countries [the men of the apparatus] are headed by a ruler, who has a personal entourage (his court) and who control and directs his numerous civil and military underlings through a corps of ranking officials. This hierarchy, which includes the sovereign, the ranking officials, and underlings is basic to all Orientally despotic regimes."

59 James Morris Blaut,Eight Eur `centric hist 'rians, Guilford Press, 2000, p. 179-180: "We are told that private property rights are inherent in the Judeo-Christian tradition, as is the idea of a personal freedom (co-authored by the Greeks) [...]: the argument is traditional European cultural conceit."

60 Sheldon S. Wolin, Dem 'cracy Inc 'rp 'rated: Managed Dem `cracy and the Specter 'f Inverted T`talitarianism, Princeton University Press 2008, p. 242.

61 François Jullien, Les atm`sphères de la p`litique. Dial`gue p`ur un m`nde c`mmun,ed. Bruno Latour \& Pasquale Gagliardi, Les Empêcheurs de tourner en rond,Paris, 2006.

62 Dong Lisheng "Démocratie locale en Chine : quelle direction après 20 ans d'expérimentation? ", Perspectives Chin `ises, 2010, $\mathrm{n}^{\circ} 2$.

63 Yong Deng, China's struggle f' $r$ status: the realignment ' $f$ internati 'nal relati ' $n s$, Cambridge University Press, 2008, 256-7: "As the influential Chinese scholar Wang Jisi observed, 'There is the notion among many Chinese that the revival of the Chinese nation would not be meaningful and real if the mainland failed to achieve reunification with Taiwan'."

64 Melissa J. Brown, Is Taiwan Chinese?: The Impact 'f Culture, P'wer, and Migrati 'n 'n Changing Identities, Berkeley, University of California Press, 2004, p. 22: “... 'if Taiwan's people are culturally Han, they should be a part of the nation of China' [...] Several assumptions about Han ethnic and Chinese national identities that are built into this idea need to be examined. First Han ethnic identity is linked to Chinese national identity. Second, Chinese national identity is linked to Han culture. Third, Chinese national identity has a clear border, and a person or group is located on one side of it or the other; that is a person or group is definitively Chinese or not Chinese, Han or not Han. All these assumptions are problematic."

65 Jacques deLisle, "Democratization in Greater China: Introduction", Orbis, Spring 2004, 48(2): 193-203: "Taiwan's rapid democratization began in the late 1980s and extended through several national elections, including a presidential election in 2000 that produced a peaceful transfer of power to the opposition party and another, in 2004, that brought a close and heated contest between the former opposition party's incumbent and the former ruling party's challenger". 
66 Fiorella Allio, « Démocratisation et processus électoral à Taiwan », in La Chine et la Dém `cratie, Mireille Delmas-Marty et Pierre-Etienne Will (Dir.), Fayard, 2007, pp. 735-801 : " La démocratie n'est pas un mode de gouvernance allant de soi et fonctionnant sur commande ; elle est bien plutôt le fruit d'une évolution historique, et l'on sait que sur ce plan la situation de l'île, interne et externe, est trop complexe pour qu'une telle évolution procède de façon linéaire et sans rencontrer d'embûches. Le bilan prudent que nous proposerons [est que la] démocratie taïwanaise est toujours en phase de 'normalisation'. "

67 Laurence Whitehead, "The Democratization of Taiwan: a comparative perspective", Dem 'cratizati ' $n$ in Taiwan: implicati ' $n s f^{\prime} r$ China, Steve Yui-Sang Tsang, Hung-mao Tien, eds., MacMillan Press 1999, p. 168-187.

68 Vermander, Benoit, " Chine - Taiwan », Études, mars 2009, n 4103 : « il est possible que le rapprochement avec la Chine et l'érosion des libertés publiques deviennent synonymes dans l'esprit de nombreux citoyens. »

69 Susan Shirk, China: fragile superp `wer, Oxford University Press, 2007, p. 204: "We [selon les propos anonymes d'un intellectual chinois rapportés par Susan Shirk] have suffered internally and externally because of the unsolved issue of Taiwan. We have had to give too much to the U.S. only to hear the words 'one China'. We will have more leverage if the Taiwan issue disappears. We need to get rid of this burden, rather sooner than later. It would unify society. China has robust domestic demand. It can withstand war and sanctions."

70 Baogang He, "China's national identity: a source of conflict between democracy and state nationalism”, Nati 'nalism, dem `cracy and nati 'nal integrati $n$ in China, Leong H. Liew, Shaoguang Wang eds., Routledge 2004, pp. 170-195.

71 Stéphane Corcuff, " Le Kuomintang, parti nationaliste taïwanais ? Quelques réflexions sur le «paradoxe identitaire » à Taiwan »Sens Public, Avril 2008 : « ce qu'il faut bien appeler un impérialisme : culturel, politique, et surtout ethniciste, celui d'une Chine qui refuse, avec une perception absolutiste et sacrée de 'l'unité', toute solution moderne de recomposition politique des territoires chinois et sinisés sur le mode, par exemple, fédéral ».

72 Pierre Vidal-Naquet, « Histoire et idéologie : Karl Wittfogel et le concept de 'mode de production asiatique' ",Annales. Éc 'n `mies, S`ciétés, Civilisati 'ns, 1964, vol. 19, $\mathrm{n}^{\circ}$ 3,pp. 531-549: «Mais Montesquieu lui-même, s'il a donné une forme quasi-définitive au mythe du despotisme oriental, dérive d'une longue tradition dont on peut faire l'histoire depuis Hérodote et Aristote : le desp `tès est le propriétaire d'esclave ; et le despote que, dès la fin du Moyen Âge, on oppose au souverain chrétien est le roi oriental ; tradition qui, de Jean Bodin à la fin du règne de Louis XIV prend une signification politique de plus en plus précise. »

73 Chia-Lung Lin, "The political formation of Taiwanese nationalism”, Nati nalism, dem 'cracy and nati 'nal integrati ' $n$ in China, Leong H. Liew, Shaoguang Wang eds., Routledge 2004, pp. 122-144. "The official name of Taiwan is still Republic of China, but the exercise of democracy in the island, especially direct presidential elections, may be seen as a form of self-determination that proves Taiwan's independent sovereignty."

74 Voir la critique nuancée de A. C. Graham, Studies in Classical Chinese Phil `s `phy and Phil `s `phical Literature, 1990, University of New York Press, p. 329-330: "Are we to conclude that only Indo-European languages have a word for the concept of Being, and that other languages are forced to fill the gap with words that really mean 'have', 'this' and 'make' ? The absurdity of this conclusion becomes apparent as soon as it is puts into words; ... as a hidden presupposition, it may be fatal to understand of Chinese discussions of shih/fei and $y u / w u . . . "$

75 Pour une critique de cette affirmation, on pourra se rapporter à Qiang Fang, Roger des Forges, "Were Chinese Rulers above the Law: Toward a Theory of the Rule of Law in China from Early Times to 1949 C.E.” Stanf 'rd J'urnal 'f Internati 'nal Law, $\mathrm{n}^{\circ} 44.1$ (spring, 2008): 101-146.

76 Pour les mathématiques, voir la mise de point de Katherine Chemla, « Penser sur la science avec les mathématiques de la Chine ancienne », in Anne Cheng (éd.), La pensée en Chine auj 'urd'hui. Gallimard, Folio, 2007, p. 353-386.

\section{Pour citer cet article}


Référence électronique

Jean-Yves HEURTEBISE , « De la Démocratie en Chine : éssai d'archéologie du

discours sinologique », Transtext(e)s Transcultures 跨文本跨文化 [En ligne] , 6 | 2011 , mis en ligne le 05 avril 2011, Consulté le 26 avril 2011. URL :

http://transtexts.revues.org/index404.html

\section{Auteur}

\section{Jean-Yves HEURTEBISE}

Jean-Yves Heurtebise, docteur de philosophie, est membre du CRAL (EHESS - UMR 8856), membre associé du CEPERC (Université de Provence, Aix-Marseille 1 - UMR 6059) et co-fondateur du Kozmestky Global Collaboratory's Center for Everything (dédié à la résorption du "knowledge gap" et à la fondation d'une pratique académique durable) de Stanford University. De 2009 à 2011, dans le cadre du programme européen STF-China, il a été chercheur invité à la National Taiwan University. Entre 2011 et 2012, il sera chercheur invité à Tsing Hua University (Chine), dans le cadre d'un projet international (Marie Curie IRSES) d'une durée de trois ans comportant neufs universités (Université de Turin, Université de Lund, Université de Tomsk, Université de Tsing Hua, Université de Provence, etc.). Ses recherches sont dédiées à l'étude des rapports entre Culture (linguistique, sociologie, esthétique) et Catégories de l'esprit : peut-on mettre en évidence l'existence de matrices différentielles de la production d'idées échappant autant à l'homogénéité de l'Universel qu'à l'opposition systématique du Culturalisme ? Cette problématique centrale est développée autour de trois axes de recherche : esthétique (Jean-Yves Heurtebise, « Penser la danse ou qu'est-ce qu'un Corps ? (Un Univers Merleau-Pontien) », in S.-A. Crevier-Goulet (Dir.), Penser les matières du corps, Paris, Sorbonne Nouvelle, 2011, pp. 219-232), épistémologique (Jean-Yves Heurtebise, « Le Temps est-il une qualité seconde ou première du Réel ? Science et Philosophie d'Aristote à Bergson », Philosophia Scientiae, 15 (3), 2011) et comparatiste (Jean-Yves Heurtebise, « Essai d'une géographie symbolique de la morale », Le Portique, $\left.n^{\circ} 5,2007\right)$.

Dr. Jean-Yves Heurtebise holds a PhD in Philosophy. He is a statutory member of the CRAL (EHESS), an associate member of the CEPERC (University of Provence) and co-founder of Stanford University KGC's Center for Everything (aiming at filling the knowledge gap through the implementation of sustainable scholarship). Between 2009 and 2011, sponsored by the European Commission program Science and Technology Fellowship in China, he was Visiting Scholar in the Philosophy Department of National Taiwan University. Between 2011 and 2012, he will be Visiting Scholar in Tsing Hua University, in a three years international collaborative project (Marie Curie IRSES) gathering nine institutions (Turino University, Lund University, Tomsk University, Tsing Hua University, Provence University, etc.). His research is dedicated to the exploration of the relationships between Culture and Categories and tried to pave the way of a new paradigm for Comparative Studies avoiding both Mind Universalism and Cultural segregationism. Inside this main flow, three different sustained currents of analysis: aesthetics (Jean-Yves Heurtebise, "From Tarde to Superman: Ordinary Heroism and Superheroes: an American History", Glimpse, n`9, Proceedings of the Society for Phenomenology and Media, 2011, pp. 68-78), epistemology (Jean-Yves Heurtebise, "Deleuze's philosophy and the biology of Apoptosis", in L. Cherlonneix \& J.-C Ameisen (Eds.), New representations of Life in Biology and Philosophy, Paris, de Boeck, 2011) and comparative studies (Jean-Yves Heurtebise, "History of cultural relationships between Europe and China: myths and prejudices", in Juan Yao-chueh (Ed.), FrancoChinese Cultural Dialogues, Taipei, National Central University Press, 2011 (in press)).

\section{Droits d'auteur}

(C) Tous droits réservés 